\title{
Recognition memory for sentences of varying length
}

\author{
A. J. WEARING, Yale University, New \\ Haven, Conn. 06510
}

Subjects learned 40 sentences, 10 each of $11,12,13$, or 14 words in length. Either immediately or $24 \mathrm{~h}$ later they were given a recognition test. Neither retention interval nor sentence length produced any significant differences.

The effects of sentence length on sentence learning have excited curiously little interest among students of word strings. It is not even clear how much it is necessary to vary sentence length in order to produce variations in retention, since a sentence is not made up of independent words, but consists of variable-size chunks

The problem of length differences can be sidestepped in research by either dismissing them as relatively unimportant (Fodor \& Garrett, 1966, pp. 146-147), or by using only sentences of identical length. However, the second control is not always possible. For example, in any particular sentence the passive usually contains about two more words than the active voice. There is a considerable literature showing that passive sentences are more difficult to learn than active ones (Garrett \& Fodor, 1968), but it is at least possible that this supposedly well established finding is in part an artifact of sentence length, since when Martin \& Roberts (1966) used truncated passives (thus making equal the length of their various sentences) they found no difference between active and passive sentences.

An oft-cited experiment carried out by Savin \& Perchonock (1965) confounded sentence length and transformational complexity. Ss were presented with a sentence plus a list of unrelated words, and immediately had to recall the sentences and as many words as possible. The number of unrelated words successfully recalled was presumed to be a measure of the storage requirements for a particular sentence type. From their results the authors concluded that the storage required depended on the number of Table 1

transformations involved, but as can be seen from Table 1, the confounding of sentence length with transformational complexity also permits the conclusion that the observed differences were simply due to differences in the number of words recalled.

Martin \& Roberts (1967) explicitly investigated the effect of differences in sentence length by comparing the free recall of five-, seven-, and nine-word sentences that were syntactically similar. They found highly significant differences between the three lengths, with the greatest difference (larger by a factor of two) being between the seven- and nine-word groups.

The sharp decline from seven- to nine-word sentences found by Martin \& Roberts (1967) is also evident in Savin \& Perchonock's data (see Table 1). This finding suggests that immediate memory capacity is an important variable in processing shorter word strings. The other side of the coin is that findings of subcapacity sentences may not be true of longer ones. There is, for example, some evidence that the perception of the location of a click in listening to sentences is independent of phrase structure for 5-word sentences, although not for 12-word ones.

The aim of the present study was to consider the question of memory for longer sentences and extend the findings of Martin \& Roberts (1967) by examining recognition memory for $11-, 12-, 13-$, and 14-word sentences over two retention intervals, 0 and $24 \mathrm{~h}$. The procedure involved presenting $S$ with 44 sentences during the acquisition phase of the experiment, and then giving him a recognition test in which 42 of the same 44 sentences were presented, intermixed with 42 new sentences. During the test the sentences were presented one at a time, and Ss had to judge whether or not each sentence was old (seen at acquisition) or new.

\section{METHOD}

Acquisition Sentences

These consisted of four groups of 10 1

Relation between sentence length and number of additional unrelated words recalled in Savin's and Perchonock's experiment

\begin{tabular}{ccc}
\hline Sentence Length & Transformations & Mean number of Additional words recalled \\
\hline 5 & Wh & 4.78 \\
6 & K, Q, E & 4.88 \\
7 & N, QN & 4.42 \\
8 & P, PQ, EP & 4.08 \\
9 & NP, NPQ & 3.67 \\
\hline
\end{tabular}

sentences each, with 4 additional sentences to provide buffer items at the beginning and the end of the list. The four groups differed in the number of words per sentence-11, 12, 13, or 14 words, respectively.

None of the sentences shared any common content words (nouns, adjectives, verbs, and adverbs), and word frequency was equated across groups. The numbers of letters in every sentence were approximately equal. Each sentence was active and declarative, possessing a subject, object, verb, and adverbial phrase. Typical sentences were:

(1) The glib salesman persuaded the young housewife in the dining room.

(2) The tedious nobleman asserted his undying desire with a passionate moan.

(3) The big weight lifter thrust his hairy chest out with unusual lack of effort. Distractor Sentences

The distractor sentences were obtained by taking each acquisition (or old) sentence and altering it either by changing the position of the adverb, changing an adjective to an adverb, or vice versa, or by moving the adverbial phrase to another position within the sentence. Thus the distractor sentences for the examples given above were:

(4) The glib salesman in the dining room persuaded the young housewife.

(5) The nobleman tediously asserted his undying desire with a passionate moan.

(6) The big weight lifter thrust out his hairy chest with unusual lack of effort.

Distractors were chosen so that each type of distractor appeared equally often in each group.

For each acquisition or "old" version of a sentence, there was a corresponding distractor of "new" version, and the terms old and new will be used hereafter to refer to different versions of the same sentences. Experimental Design

All the sentences were learned by every $\mathrm{S}$; then one group of $11 \mathrm{Ss}$ was given a recognition test immediately after acquisition, while a second group of $7 \mathrm{Ss}$ was tested after a retention interval of $24 \mathrm{~h}$.

The presentation sequence at acquisition began and finished with two buffer sentences, included to reduce any possible primacy-recency effects. Sequences for the intervening 40 sentences was obtained by randomly choosing a sentence from a randomly chosen length group with the constraint that each group had to be chosen exactly once in the first four choices, as well as for all successiv z groups of four choices. Every $S$ had a different sequence at acquisition. 
For the recognition test another sequence of acquisition or old sentences was generated, following the rules described in the previous paragraph. Distractor or new sentences were then randomly interleaved between the old sentences with the constraint that no more than three old or new sentences could appear consecutively. A second constraint was that for half the sentences the new version appeared second. $A$ third constraint was that at least 25 sentences intervened between the appearance of the old and new versions of a sentence.

\section{Procedure}

Subjects were run on the University of Illinois PLATO system (Bitzer, Hicks, Johnson, \& Lyman, 1967). Each S sat at a booth with a TV screen and typewriter keyset which were connected with a computer. Instructions, stimuli, and responses appeared on the screen and Ss typed their responses on the keyset.

When the Ss were comfortably seated at their terminals they were presented with three practice acquisition sentences. They were given $31 \mathrm{sec}$ to write down each sentence on a prepared form which was next to their keyset. The writing out of the sentences was to ensure that $S$ s paid approximately the same amount of attention to every word in the sentence.

Following the practice sentences a list of 44 sentences was presented, comprising the 40 experimental sentences preceded and followed by two buffer sentences. As in the practice trial each sentence was shown for $31 \mathrm{sec}$. Three seconds before the end of each presentation, the words "three seconds left" were displayed on the screen.

At the retention test either immediately or $24 \mathrm{~h}$ later the $\mathrm{Ss}$ were presented with a list of six practice sentences composed of the three practice sentences learned at acquisition and three new (distractor) sentences. Each sentence was shown for $11 \mathrm{sec}$, and for each sentence the Ss had to indicate whether or not the sentence was old or new by pressing appropriately labelled keys

Following the practice trial Ss were presented with the test list proper: four buffer sentences followed by the 0 test sentences.

\section{Subjects}

Eighteen paid undergraduate men served as Ss.

RESULTS AND DISCUSSION

The number of correct old sentences per
Table 2

Mean number of old sentences correctly recognized in each group (number of words in each cell expected correct by chance is five).

\begin{tabular}{|c|c|c|c|c|c|}
\hline \multirow[t]{2}{*}{ Retention Interval } & \multicolumn{4}{|c|}{ Sentence Length (Words per Sentence) } & \multirow[t]{2}{*}{ Mean } \\
\hline & 11 & 12 & 13 & 14 & \\
\hline 0 hours & 7.64 & 8.00 & 7.82 & 7.14 & 7.66 \\
\hline 24 hours & 7.14 & 7.00 & 8.14 & 6.71 & 7.25 \\
\hline Mean total & 7.44 & 7.61 & 7.94 & 7.00 & \\
\hline
\end{tabular}

cell was analyzed with a three-way repeated measures analy sis of variance. The results are summarized in Table 2. For retention interval, $F(1,16)=1.07$, for sentence length (in words), $F(3,48)=1.23$, and for retention interval by sentence length, $F(3,48)=0.57$. None of these ratios is significant $(p>.25)$

One problem with experiments of this kind is that of response bias, that is, differences in the tendencies of individuals to respond either "old" or "new." One S, for example, may be willing to regard any sentence as old, unless he is absolutely sure that he did not see the sentence before. Another $S$ may adopt exactly the reverse position. Fortunately, the use of a repeated measures design means that any particular S's bias is distributed across all levels of the within-Ss variable. It is necessary to assume, of course, that Ss did not detect the differences in sentence length, and respond differentially to sentences, depending on the number of words that they contained. Questioning of the Ss after the experiment revealed no evidence of any differential response bias, and indeed only one $S$ was able to say that the sentences did, indeed, differ systematically in the number of words that they contained.

A second way to examine the assumption of consistent within-S response bias is to look at the number of old and new sentences that individual Ss got correct, at each level of sentence length. Chi squares on the distribution of old/new responses across levels of sentence length were computed for each $S$ and none was significant at the $5 \%$ level, which allows us to conclude that the Ss' biases were reflected about equally at all levels of the within-Ss factor.

From these results it may be concluded that although small differences in the number of words in sentences are important in the free recall of sentences of 9 or less words (Martin \& Roberts, 1967), they do not matter for longer sentences of 11 or more words in length in a recognition test.
Since the present experiment differed from that of Martin and Roberts with regard to number of words per sentence and type of retention test, the reason for the different results cannot be laid to any single cause.

However, it does seem as though sentence length does not interact in any simple way with learning difficulty, and that it is possible that long and short sentences may be coded and stored differently. The present results do not allow any speculation on the nature of that difference. What one may conclude from the foregoing considerations is that experiments on memory for sentences should take more seriously than they have in the past the effects of variables like sentence length and immediate memory load.

\section{REFERENCES}

BITZER, D., HICKS, B., JOHNSON, R., \& LYMAN, E. R. The Plato system: Current research and developments. IEEE Transactions on Human Factors in Electronics, 1967, 8 , 64-70.

FODOR, J. A., \& GARRETT, Mr. Some reflections on competence and performance. In J. Ly ons and R. J. Wales (Eds.), Psycholinguistic papers. Edinburgh: Edinburgh University Press. 1966.

GARRETT, M., \& FODOR, J. A. Psychological theories and linguistic constructs. In T. R. Dixon and D. L. Horton (Eds.), Verbal behavior and general behavior theory. Englewood Cliffs, N.J.: Prentice-Hall, 1968. Pp. 451-477.

MARTIN, E., \& ROBERTS, K. H. Grammatical factors in sentence retention. Joumal of Verbal Learning \& Verbal Behavior, 1966, 5, 211-218.

MARTIN, E., \& ROBERTS, K. H. Sentence length and sentence retention in the free learning situation. Psychonomic Science, 1967, 8, 535-536.

SAVIN, H. B., \& PERCHONOCK, E. Grammatical structure and the immediate recall of English sentences. Joumal of Verbal Learning and Verbal Behavior, 1965, 4, 348-353.

\section{NOTES}

1. I wish to thank Clinton Walker, who criticized an earlier draft of this paper and made substantive suggestions for its improvement, and Rosemary Wearing. who assisted in the preparation for and the running of the experiment.

2.C. Walker, personal communication. 